\title{
ECOLOGIA DE TRIATOMINNEOS E TRANSMISSÃO DO TRYPANOSOMA CRUZI, COM ESPECIAL REFERENCIA AO BRASIL
}

\author{
Mauro Pereira Barroto
}

Com base em seu comportamento ecológico e seu grau de relacionamento com o homem e os mamiferos domésticos, comensais e sinantrópicos, os triatomíneos são distribuidos em seis grupos:

1) Triatomíneos tipicamente silvestres, isto é, espécies só encontradas em ecótopos naturais e que nunca freqüentam as habitações humanas e suas dependências. Conseqüentemente, nunca entram em contacto com o homem e os mamiferos domésticos, a não ser acidentalmente quando estes penetram nos focos naturais. Entretanto, podem ter papel maior ou menor na manutenção da enzootia tripanossômica silvestre. Exemplos: Psammolestes coreodes, Psammolestes tertius, Cavernicola pilosa, Triatoma dispar, Triatoma delpontei e muitas outras espécies cujos hábitos são pouco conhecidos.

2) Triatomíneos essencialmente silvestres cujos adultos invadem, com maior ou menor freqüência, as habitações humanas e suas dependências, sem, todavia, aqui se colonizar. Além do papel que têm no ciclo silvestre de transmissão do T. cruzi, podem, ao entrar em contacto com o homem e os mamíferos domésticos e domiciliados suscetíveis, transmitir-lhes a infecção, tanto em áreas silvestres quanto em ecótopos artificiais. Exemplos: Panstrongylus geniculatus, Triatoma rubrovaria, Triatoma arthumeivai, Triatoma patagonica, Triatoma eratyrusiforme, Rhodnius domesticus e muitas outras espécies cujos hábitos são poucos conhecidos.

3) Triatomineos silvestres em fase inicial de adaptação aos ecótopos artificiais, formando pequenas colônias principalmente no peridomicílio e, mais raramente, na própria habitação humana. Além da importância que têm no ciclo silvestre do T. cruzi, podem trazer a infecção para os ecótopos artificiais $e$, em determinadas instâncias, participar do ciclo domiciliário do parasita. Exemplos: Rhodnius neglectus, Triatoma vitticeps, Triatoma platensis e outras espécies pouco estudadas.

4) Triatomíneos que se criam indiferentemente em ecótopos naturais e artificiais. Embora tenham conseguido adaptar-se com maior ou menor sucesso à habitação humana e suas dependências, são encontradas também em diferentes ecótopos naturais. Além de participar do ciclo silvestre do T. cruzi têm importância no transporte do parasita para os ecótopos artificiais e na infestação inicial ou na reinfestação destes ecótopos livres de insetos pelo expurgo, constituindo também elos na cadeia de transmissão domiciliária da infecção. Exemplos: Panstrongylus megistus, Rhodnius prolixus, Rhodnius pallenscens, Triatoma sordida, Triatoma brasiliensis, Triatoma maculata, Triatoma pseudomaculata, Triatoma quasayana etc. Departamento de Ciências Patológicas, Disciplina de Parasitologia, da Faculdade de Farmácia e Odontologia de
Ribeirão Preto, da USP.

Recebido para publicação em 18-8-1976. 
5) Triatomíneos bem adotados aos ecótopos artificiais, mas, às vezes, ainda encontrados em focos residuais naturais. São os insetos mais importantes no ciclo domiciliário do T.cruzi, mas podem, pelo menos em certas áreas, participar do ciclo silvestre da infecção. Exemplo: Triatoma infestans.

6) Triatomíneos estritamente domiciliados. Apesar disto, parecem ter menor importância no ciclo domiciliário, especialmente na transmissão do T.cruzi ao homem, porque convivem mais com ratos comensais, embora também entrem em contacto com o homem. Exemp/o: Triatoma rubrofasciata.

A tripanossomose americana, era, primitivamente, uma enzootia, afetando exclusivamente animais silvestres e sendo transmitida por triatomíneos também silvestres. Com o correr dos tempos tornou-se uma zoonose, quando o homem entrou em contacto com os focos naturais, alterou o equilíbrio ecológico e possibilitou a domiciliação de algumas espécies de insetos que, dessa maneira, carrearam o T.cruzi para os ecótopos artificiais, isto é, para as habitações humanas e suas dependências.

Essa domiciliação, ao que tudo indica mais antiga nas regiões andinas e mais recente na parte leste da América do Sul, foi um fenômeno lento, como todo processo de seleção natural. $E^{E}$ difícil apontarem-se provas diretas desse fenômeno, mas, como assinala Lucena ${ }^{64}$, sua história pode ser reconstituída através da observação dos vários graus de adaptação de diferentes espécies aos ecótopos artificiais.

A maioria dos triatomíneos conserva seus hábitos silvestres primitivos. Embora, muitas vezes, sejam encontrados com infecção natural, esses triatomíneos raramente ou nunca chegam a entrar em contacto com o homem e, por isso, ainda que tenham papel destacado no ciclo silvestre do T.cruzi, são considerados de pequena importância epidemiológica para o homem. No extremo oposto estão as espécies domiciliadas ou sinantrópicas que vivem em íntimo e diuturno contacto com o homem e que, por isso, são responsáveis pelo ciclo domiciliar da infecção e são consideradas as mais importantes do ponto de vista epidemiológico. Entre os dois extremos há toda uma série gradual de transição. Assim, pois, é difícil fazer uma classificação ecológica dos triatomíneos, sendo essa dificuldade aumentada pela escassez ou mesmo inexistência de dados sobre o comportamento de numerosas espécies, algumas das quais conhecidas apenas pelos tipos ou alguns exemplares de museus.
Apesar dessas limitações e tendo presente que a domiciliação é um fenômeno essencialmente dinâmico e em vias de realização, podemos, tentativamente, distribuir os triatomíneos, segundo suas relações atuais com os ecótopos naturais e artificiais, em seis categorias. Seja dito de passagem que essas categorias são, em linhas gerais, aquelas aceitas por Zeledon ${ }^{95,96}$ e divergem completamente das estabelecidas por Miles ${ }^{71}$.

\section{TRIATOMINEOS TIPICAMENTE SILVES- TRES}

Este grupo inclui aquelas espécies que, até agora, têm sido encontradas exclusivamente em ecótopos silvestres, nunca freqüentando, mesmo na forma adulta alada, as habitações humanas e suas dependências, ainda que estas estejam localizadas nas vizinhanças daqueles ecótopos. Esses triatomineos alimentam-se em animais silvestres, geralmente mam fferos e aves, e nunca entram em contacto com o homem e os animais domésticos, a menos que estes penetrem nos focos naturais.

Exemplos característicos desse grupo são as espécies do gênero $P$ sammolestes, especialmente - P.coreodes e o P.tertius que têm sido mais estudados. O P.coreodes ocorre na Argentina, Paraguai, Bolívia e Oeste do Brasil ${ }^{62}$. Na Argentina habita ninhos de pássaros vários, entre os quais os de Myopsitta monacha, segundo Abalos e Wygodzinsky ${ }^{2}$ e Abalos ${ }^{1}$; no Brasil é encontrado em ninhos de Phacellodomus rufifrons sincipitalis, segundo Lent ${ }^{58} \mathrm{e}$ Barreto $^{12}$, alimentando-se exclusivamente em aves, como mostram as reações de precipitina ${ }^{1} 1$. O P.coreodes nunca foi encontrado com infecção natural e, assim, parece não ter papel nem mesmo no ciclo silvestre de transmissão do T.cruzi. Já o P.tertius, nas regiões Leste e Nordeste do Brasil, habita quase exclusivamente 
ninhos dos furnarfidas, Anumbius annumbi, Phacellodomus rufifrons, rufifrons e Phacellodomus rufifrons specularis, segundo Corrêa, Carvalheiro e Barreto ${ }^{31}$, Lucena e Lucena ${ }^{65}$, Lent e Jurberg ${ }^{6} \dot{i}$, Barreto e Carvalheiro $^{16,17,18}$, e Barreto e Albuquerque ${ }^{14}$. Alimenta-se principalmente de sangue de aves, mas também suga ratos e gambás, quando estes passam a ocupar os ninhos velhos abandonados por seus construtores ${ }^{17}$. A infecção experimental e natural pelo T.cruzi foi registrada por Barreto, Albuquerque e Funayama ${ }^{15}$ em 1969 $\epsilon$ Barreto e Albuquerque ${ }^{14}$ em 1969. Embora os achados de exemplares com infecção natural sejam pouco numerosos o P.tertius pode ser considerado um elo, ainda que secundário, no ciclo silvestre da transmissão do T.cruzi.

Outro triatomíneo tipicamente silvestre é a Cavernicola pilosa, até agora só encontrada em ocos de árvores onde vive associada a morcegos. Essa associação foi observada por Dias, Mello, Costa, Damasceno e Azevedo ${ }^{42}$ em 1942, no Pará e, mais recentemente, confirmada por Barreto $^{11}$ em 1971, no Sul de Mato Grosso. Este último autor capturou exemplares com sangue de morcego no tubo digestivo, demonstrando pelas reações de precipitina; alguns exemplares estavam infectados pelo T.cruzi. Assim, a C.pilosa é um dos vectores em jogo no ciclo silvestre de transmissão do T.cruzi entre morcegos.

Outra espécie tipicamente silvestre é o Triatoma dispar do Paraná. Tem hábitos arboríco$\operatorname{las}^{48}$, vivendo associado à preguiça, Choloepus hoffmanni. Sua infecção natural foi recentemente observada por Sousa e Galindo ${ }^{86}$ que sugerem que o triatomíneo em questão desempenha importante papel vector entre animais arborícolas. Releva assinalar que exemplares de T.dispar são atraídos por isca humana na copa das árvores e, assim, segundo Sousa e Galindo $^{86}$, pode se envolver ocasionalmente na transmissão do T.cruzi ao homem, quando este entra nos focos naturais no Panamá.

Também tipicamente silvestre é o Triatoma delpontei, na Argentina, que habita exclusivamente ninhos de Myiopsitta monacha. Segundo Abalos ${ }^{1}$, a infecção natural desse triatomíneo já foi registrada, o que se explicaria pela presença de roedores parasitados nos ninhos daquele pássaro. Sucede, assim, com o T.delpontei aquilo que se observa com o P.tertius, isto é, a espécie em questão pode ser considerada como elo, ainda que secundário, no ciclo silvestre do T.cruzi.
Outra espécie também silvestre é o Triatoma garciabecei que, na Argentina, se coloniza em ninhos de pássaros e sob cascas parcialmente destacadas de árvores, nunca evadindo ecótopos artificiais ${ }^{1}$. Sua infecção natural foi observada por Martinez e Cichero ${ }^{68}$, em 1972, e, assim, o triatomíneo constitui um elo no ciclo silvestre de transmissão do T.cruzi.

Talvez possam ainda ser incluídos no grupo de triatomíneos exclusivamente silvestres as espécies de Microtriatoma, Belminus, Parabelminus, Dipetslogoaster, assim como espécies palmicolas de Rhodnius sobre as quais faltam informações mais detalhadas.

\section{TRIATOMINEOS SILVESTRES CUJOS ADULTOS INVADEM ECÓTOPOS ARTIFI- CIAIS}

Este grupo inclui triatomíneos essencialmente silvestres cujos adultos, com maior ou menor freqüência, invadem habitações humanas e suas dependências sem, contudo, colonizar-se nelas. Essa invasão se dá por atividade própria do inseto alado talvez motivada pela atração exercida pela luz. Além do papel importante que têm no ciclo silvestre de transmissão do T.cruzi, podem, ao entrar em contacto com o homem e os animais domésticos e domiciliados suscetíveis, transmitir-lhes a infecção nos ecótopos artificiais.

Um exemplo típico é oferecido pelo Panstrongylus geniculatus. Espécie de ampla distribuição na Região Neotropical, cria-se exclusivamente em locas de animais, especialmente buracos de tatus; nesses ecótopos apresenta altos índices de infecção, como demonstram as observações de Barreto ${ }^{8}$, e é, assim, importante elo no ciclo de transmissão do T.cruzi entre aqueles desdentados, segundo Barreto ${ }^{10}$. Exemplares adultos, muitos dos quais infectados, têm sido capturados em habitações humanas e anexos. Assim, na Venezuela, Guerrero, Garcia e Domingues $^{55}$ registraram Indices de infecção de $10 \%$ entre adultos capturados dentro de casa. Barreto ${ }^{13}$ em 1975, no Brasil, capturou no interior de uma habitação rural, exemplares, alguns dos quais infectados com sangue humano e de cão no tubo digestivo, identificado pela reação de precipitina. Isso mostra que nos ecótopos artificiais o P.geniculatus pode entrar em contato com o homem e animais domésticos susce tiveis para obter alimen to e, assim, transmitir-Ihes a infecção. 
Outra espécie considerada tipicamente silvestre é o Triatoma rubrovaria, no Sul do Brasil, no Uruguai e no Norte da Argentina. Seus criadouros primitivos são buracos e fendas em lugares pedregosos, onde convive com roedores $e$ onde pode apresentar altos índices de infecção, sobretudo na Argentina ${ }^{1}$. Mas o T.rubrovaria se cria também em muros e cercas de pedras construídas pelo homem ${ }^{82}$. Apesar disso, Talice Costa, Rial e Osimani ${ }^{87}$ e Pinto ${ }^{80,81}$ o consideram essencialmente silvestres e asseveram que ele não se coloniza em habitações humanas, fato que parece corroborado pelas verificações de Coutinho, Pinto e Barbosa ${ }^{40}$ e Abalos $^{1}$. Não resta, porém, dủvidas de que exemplares adultos, principalmente nos meses de verão, invadem as habitações humanas, onde exemplares infectados são encontrados ${ }^{83}$. Quanto à sua eventual antropofilia, ela parece demonstrada pelas observações de Toranzos ${ }^{88} \mathrm{e}$ Abalos ${ }^{1}$. Assim, além de ser um elo no ciclo silvestre de transmissão do T.cruzi, o T.rubrovaria pode entrar em contacto com o homem, tanto nos focos naturais quanto no interior das habitações, transmitindo-lhe a infecção.

Outro exemplo de espécie tipicamente silvestre é oferecido pelo Triatoma arthurneivai no Brasil. Seus criadores naturais são buracos e fendas em lugares pedregosos, onde vive associado a lagartos, especialmente Tropidurus torquatus, e, mais raramente a roedores, segundo as observações de Lent e Martins ${ }^{63}$, Barreto $^{6}$, Corrêa, Alves e $\mathrm{Noda}^{34}$, Forattini, Juarez é Rabello ${ }^{45}$. Tanto no Estado de Minas Gerais como no Estado de São Paulo, adultos têm sido capturados em habitações humanas $e$ anexos, segundo os dados de Pellegrino ${ }^{76}$, Corrèa, Alves e Pascale ${ }^{33}$, Alves e Noda ${ }^{4}$, Forattini, Juarez e Rabello $^{45}$. Embora sua infecção experimental haja sido conseguida, não há registro de infecção natural, tanto nos focos naturais quanto nos biótopos artificiais e, assim, o triatomíneo em tela parece destituído de importância no ciclo de transmissão do T.cruzi.

Outro triatomíneo deste grupo é o Triatoma eratyrusiforme das regiões serranas da Argentina, onde se cria em lugares pedregosos e convive com roedores e desdentados ${ }^{1}, 28$. Segundo Martinez e Cichero ${ }^{68}$ ele é muito abundante em certas áreas e, às dezenas, ataca os viajantes que, à noite, acampam nas proximidades de seus criadouros. Segundo Blaksley e Carcavallo ${ }^{28}$ e Abalos $^{1}$, exemplares adultos, muitos dos quais infectados, invadem as habitações humanas, aparentemente atraídos pela luz, mas não se colonizam nos ecótopos artificiais. Todavia, além de ser um elo no ciclo silvestre de transmissão do T.cruzi, pode transmitir o parasita ao homem, tanto nos focos naturais como no interior de habitações.

Pertence ainda ao grupo de que nos ocupamos o Triatoma patogonica na Argentina. Criase em lugares pedregosos, mas pode ser encontrado também em ocos de árvores e cactaceas secas ${ }^{1,2,28}$. Nos focos naturais alimenta-se em pequenos mamíferos, especialmente roedores, e ocasionalmente em aves ${ }^{28}$, mas ataca avidamente o homem e os animais domésticos quando estes entram em contacto com os focos naturais. $\hat{E}$ encontrado também no peridomicilio, segundo Brionis ${ }^{29}$, invadindo abrigos de animais, especialmente coelheiras, embora não se colonize nas habitações humanas propriamente ditas, onde só exemplares adultos são encontrados. Como é um bom vector e ataca o homem e animais domésticos, parece ter papel no transporte do T.cruzi para os ecótopos artificiais, além de ser um elo no ciclo silvestre de transmissão do parasita.

Triatomíneos silvestres, cujos adultos podem invadir os ecótopos artificiais são algumas espécies de Rhodnius, particularmente o R.domesticus na Baixada Litorânea do Brasil, e o R.pictipes das Regiões Norte e Centro-Oeste do Brasil. O R.domesticus foi originalmente descrito de exemplares capturados em habitações humanas e daf o seu nome ${ }^{74}$. Desde então, o encontro ocasional do triatoma em ecótopos artificiais tem se repetido $56,59,60,63,84$. No entanto, Leal, Ferreira e Martins ${ }^{56}$, em 1961 . na liha de Santa Catarina, mostraram que os criadouros naturais do triatomíneo são ninhos de ratos, Echimys dasithrix, em touceiras de bromélias terrestres $\mathrm{e} e m$ ocos de árvores. Recentemente Miles ${ }^{71}$, na Bahia, encontrou o R.domesticus em ninho de gambá em bromélias epífitas. Tanto em Santa Catarina quanto na Bahia verificou-se a infecção natural do triatomíneo, o que mostra que ele é um elo no ciclo silvestre de T.cruzi. Os exemplares adultos que invadem os ecótopos artificiais podem carrear a infecção para o homem e os animais domésticos e sinantrópicos. O R.pictipes tem por criadouros típicos a copa de palmeiras. Em carandás, Copernicia australis, no Sul de Mato Grosso, Barreto $^{11}$ capturou exemplares infectados alimentando-se em morcegos; no Amazonas adultos infectados têm sido encontrados no interior de embarcações e domicílios humanos ${ }^{3}$.

Provavelmente muitas outras espécies de Rhodnius, Triatoma, Panstrongy/us etc., pouco estudadas e consideradas raras, ou, às vezes só 
conhecidas pelos exemplares típicos, caem dentro desta categoria de triatomíneos silvestres cujos adultos ocasionalmente penetram nas habitações humanas e anexos.

\section{TRIATOMINNEOS SILVESTRES QUE PE-} NETRAM NOS ECÓTOPOS ARTIFICIAIS E DÃO ORIGEM A COLÔNIAS INCIPIENTES.

Este grupo inclui triatomíneos em fase inicial de adaptação aos ecótopos artificiais, formando pequenas colônias nos anexos domiciliários e, mais raramente, na própria habitação humana. São espécies, que, além de papel importante que têm no ciclo de transmissão silvestre do T.cruzi, podem carrear a infecção para os ecótopos artificiais e, em certas circunstâncias, participar mais ou menos ativamente do ciclo domiciliário do parasita.

Um exemplo sugestivo é o Rhodnius neglectus, no Vale do Rio Grande, Brasil. Seus habitáculos primitivos típicos são as copas de palmeiras de diversas espécies, segundo demonstram as observações feitas a partir do trabalho de Lent ${ }^{61}$; ar convive com várias espécies de aves e mamíferos silvestres especialmente gambás, ratos e morcegos, transmitindo a esses mamiferos o T.cruzi, como mostram as observações de Barreto ${ }^{6}, 10$. A presença de exemplares adultos, alguns dos quais infectados em habita ções humanas e anexos vem sendo, de há muito, assinalada em várias localidades dos Estados de São Paulo, Minas Gerais e Goiás, segundo mostram os dados reunidos por Barreto, Siqueira, Ferriolli e Carvalheiro ${ }^{20}$, em 1968, na literatura e obtidos em seus trabalhos de campo. Por outro lado, os trabalhos de Freitas, Siqueira e Ferreira ${ }^{4}$ confirmados e ampliados pelos de Barreto, Siqueira, Ferriolli e Carvalheiro $^{22}$ e Barretto ${ }^{10,13}$ mostram que o R. neglectus nas habitações humanas $e$ abrigos de animais domésticos pode alimentar-se no homem e em cães, gatos e ratos comensais. Mas o R.neglectus já está em fase inicial de adaptação aos ecótopos artificiais, como demonstraram as primeiras observações de Correa e Lima ${ }^{36}$, amplamente confirmadas por Carvalho e Verano ${ }^{30}$, Freitas, Siqueira e Ferreira ${ }^{47}$, Marques, Conessa, Naka mura, Santos e Santana ${ }^{67}$ e Barretto, Siqueira, Ferriolli e Carvalheiro ${ }^{22}$. Estes últimos autores encontraram colônias maiores ou menores adaptadas a diferentes ecótopos artificiais, observaram a infecção natural de triatomíneos dessas colônias e verificaram que esses insetos podem alimentar-se no homem e em mamfferos domésticos e domiciliados. Assim, o R.neglectus, além de constituir um bom vector no ciclo silvestre do T.cruzi, pode invadir as habitações humanàs e suas dependências carreando para elas a infecção tripanossômica. Demais, pode colonizar-se nos ecótopos artificiais e participar, como vector secundário, do ciclo domiciliário do parasita $^{22}$.

Outro exemplo é oferecido pelo Triatoma vitticeps. A presença de exemplares adultos, alguns dos quais infectados, vem sendo assinalada na Região Leste do Brasil, especialmente no Estado de Minas Gerais ${ }^{63}{ }^{76}$, onde a espécie é considerada silvestre ${ }^{76}$. Mais recentemente, Santos, Pinto, Almeida, Zaganelli, Carrancho e Netto ${ }^{84}$ e Pinto, Santos, Zaganelli, Almeida e Carrancho ${ }^{79}$, em várias localidades do Estado de Espirito Santo, observaram o T.vitticeps colonizando-se em diferentes ecótopos naturais e artificiais e exibindo, às vezes, al tos índices de infecção. No município de Alfredo Chaves, E.S., encontraram colónias inclusive nas camas, sob colchões, e, com base em seus achados, concluem que o T.vitticeps é o principal vector domiciliar na região.

Pertence a este grupo o Triatoma platensis de ampla distribuição na Argentina onde os criadouros naturais são ninhos de pássaros. Embora se alimente habitualmente em aves, sua infecção natural já foi assinalada e a explicação do fato reside na presença de ratos infectados em ninhos abandonados pelos pássaros construtores ${ }^{2}$. Exemplares adultos invadem habitações humanas, aparentemente atraídos pela luz, mas nelas não há colonização ${ }^{1}$. Mas, o T.platensis é encontrado no peridomićlí, onde forma colónias, sobretudo em galinheiros na zona rural, segundo Mazza, citado por Abalos e Wygodzins$\mathrm{kv}^{2}$, Blaksley e Carcavallo ${ }^{28}$, e, assim, é uma espécie que parece em vias de adaptação aos ecótopos artificiais.

Parecem também pertencer a este grupo algumas espécies norte e centroamericanas, como o Triatoma prostracta prostracta, Friatoma sanguisuga, Triatoma rubida uhleri, Triatoma lecticularius etc. segundo Zeledón $n^{95,96}$.

\section{TRIATOMINNEOS QUE SE COLONIZAM INDIFERENTEMENTE EM ECÓTOPOS NA- TURAIS E ARTIFICIAIS}

Nesta categoria incluem-se numerosas espé cies que embora tenham conseguido adaptar-se com maior ou menor éxito às habitações humanas, e suas dependências, são encontradas também em múltiplos e diferentes ecótopos naturais. 
Segundo Abalos e Wygodzinsky ${ }^{2}$, esse grupo de triatomíneos aproxima-se do homem sem entrar em contacto intimo com ele. São espécies que, sem abandonar definitivamente os ecótopos silvestres, invadem e se instalam nas edículas peridomiciliárias, especialmente galinheiros, pocilgos, currais, tulhas, paióis e depósitos que lhes oferecem abrigo e fonte de alimentação, só ocasionalmente entrando em contacto direto com o homem no interior das habitações propriamente ditas. Concordamos que isso possa acontecer com algumas espécies, mas, em muitos casos, o contato com o homem é intimo, freqüentemente e importante, como mostraremos adiante. Seja como for, essas espécies, além de participar ativamente do ciclo silvestre de transmissão do T.cruzi, tem importância no transporte do parasita dos focos naturais para os ecótopos artificiais, assim como na infestação inicial ou na reinfestação das habitações humanas e anexos em áreas livres de triatomineos ou onde os insetos domiciliados foram eliminados pelo expurgo, constituindo ainda importantes elos no ciclo domiciliário de transmissão do T.cruzi, como mostramos em trabalho anterior ${ }^{13}$.

Como exemplares de espécies desse grupo podemos citar: Panstrongylus megistus, Rhodnius prolixus, Rhodnius pallences, Triatoma sordida, Triatoma maculata, Triatoma pseudomaculata, Triatoma brasiliensis, Triatoma quasayana, Triatoma dimidiata, Triatoma phyllosoma etc.

Vejamos o caso do P.megistus. A primeira referência sobre sua ocorrência em focos silvestres se deve a Gomes ${ }^{52}$ que refere a captura de uma fêmea que no sul do Est. de São Paulo procurando sugar seu capturador em plena selva. A condição de vida silvestre do inseto é confirmada por Oliveira ${ }^{75}$ no Rio Grande do Sul.

A despeito disso, até há alguns anos, as pesquisas em ecótopos silvestres deram resultados negativos ${ }^{38}$ ou conduziram a achados esporádicos ${ }^{37,46,47}$. Os únicos achados mais numerosos se deviam a Leal, Ferreira e Mar$\operatorname{tins}^{56}$ que, na llha de Santa Catarina, encontraram exemplares infectados de $P$. megistus em ninhos de gambás, culcas e ratos situados em touceiras de bromélias terrestres e em ocos de árvores.

Em pesquisas extensivas levadas a efeito em áreas diversas de bacias hidrográficas do Rio Grande e do Rio Paranarba, Brasil, Barretto, Siqueira e Freitas ${ }^{23}$ em 1964, Barretto, Siqueira, Ferriolli e Carvalheiro 22 , em 1966, Barretto e Carvalheiro ${ }^{16}$, em 1966, Barretto $6,7,10,11$ e
Barreto, Albuquerque e Funayama ${ }^{15}$, capturaram milhares de exemplares de P.megistus em ecótopos silvestres diversos, principalmente em ocos e anfractuosidade do tronco de árvores, espaços entre raízes tabulares de árvores, touceiras de bromélias epifitas e terrestres, cavidade no solo ou rochas, tufos densos de vegetação, copas de palmeiras. Nesses ecótopos, os triatomíneos convivem principalmente com gambás e cuícas, morcegos, roedores silvestres, mustélidas e prociônidas etc, além de aves numerosas ${ }^{10}$. A infecção natural pelo T.cruzi mostrou-se freqüente $e$, às vezes, sobretudo entre insetos capturados em ninhos de gambás localizados em ocos de árvores, atingiu índices muito elevados. Assim, o P.megistus, nos focos naturais, constitui importante vector entre os mamiferos silvestres com os quais convive.

Nos focos naturais, o P.megistus soe atacar o homem que neles penetra, como foi verificado por Gomes ${ }^{52}$, e confirmado por Barretto ${ }^{13} \mathrm{em}$ 1975. Partindo dos ecótopos naturais, exemplares infectados, com freqüência, invadem os ecótopos artificiais e neles se colonizam, como demonstram as observações de inúmeros autores especialmente Freitas ${ }^{46}$, em 1963. Este assunto já foi amplamente discutido em trabalho anterior ${ }^{13} \mathrm{e}$, por isso, deixamos de entrar em maiores detalhes. Releva assinalar que, em virtude desse fato, o P.megistus pode causar a infestação inicial de habitações humanas e anexos livres de triatomíneos ou reinfestar aqueles que foram desinsetizados. Os exemplares infectados, nestas circunstâncias, podem carrear o T.cruzi para os ecótopos artificiais $^{13}$.

Nos ecótopos artificiais, pelo menos na região que vai do Vale do Rio Grande até o Nordeste do Brasil, o P.megistus prolifera abundantemente. Em certas áreas, como o vale do Rio Pardo e do Mogi-Guaçu, o triatomíneo tem certa preferência pelo periodomicilio, especialmente galinheiros e abrigos de animais domésticos, mas também invade habitações humanas e nelas se coloniza. Isso se dá principalmente onde o T.infestans é a espécie dominante como vector domiciliário; mas quando esta espécie é eliminada pela desintetização, o P.megistus, agora sem concorrente, passa a dominar no interior das habitações ${ }^{46}$. Mais para o norte, no Estado de Minas Gerais, o P.megistus procria abundantemente no interior das habitações onde atinge, por vezes, densidades elevadíssimas, e, em certas áreas, como o do Recôncavo Bahiano, é a principal espécie domiciliária, não sendo mesmo encontrada em ecótopos silves- 
tres $^{71,72}$. Nos ecótopos artificias o P.megistus se alimenta no homem, nos animais domésticos (especialmente cães e gatos), em mam(feros comensais (ratos) e mamiferos sinantrópicos (morcegos e gambás), além de aves, segundo mostram as reações de precipitina ${ }^{10,72}$; apresenta, com freqüência, indices de infecção elevados e constitui um importante elo no ciclo de transmissão domiciliário do T.cruzi.

O caso do Triatoma sordida é semelhante ao do P.megistus. Embora desde o trabalho de Torres $^{91}$ em 1915, tenha sido considerada como uma espécie em vias de adaptação às habitações humanas, raros tinham sido os achados de exemplares em ecótopos silvestres $^{52,92,93}$. Somente depois das pesquisas de Corrêa e Ferreira ${ }^{35}$ e Freitas, Siqueira e Ferrei$\mathrm{ra}^{47}$, complementadas pelas de Barretto, Siqueira, Ferriolli e Carvalheiro 22 , Barretto e Carvalheiro $^{46}$, Barretto $6,9,10,11$ e Barretto, Albuquerque e Funayama ${ }^{15}$ é que se confirmou a opinião de Torres ${ }^{91}$.

Essas pesquisas vieram demonstrar que o T.sordida, nos focos naturais, é ainda mais ubiquista ou euritópico que o P.megistus, colonizando-se com maior ou menor facilidade nos mais diversos ecótopos. Nesses ecótopos é também mais euritrófico que o P.megistus, convivendo com gambás e cuícas, ratos e outros roedores silvestres, morcegos e carnívoros, além naturalmente de aves, como demonstram as reações de precipitina ${ }^{10}$. A infecção pelo T.cru$z i$ é freqüentemente observada, atingindo, às vezes, indices elevados, sobretudo entre os exemplares capturados em ninhos de gambás, ratos e morcegos localizados em ocos de árvo$\mathrm{res}^{9}, 10$. Assim, o T.sordida é um importante elo no ciclo de transmissão do T.cruzi entre os mamíferos silvestres com os quais convive.

Partindo dos focos naturais, o T.sordida pode invadir os ecótopos artificiais, como já discutimos detalhadamente em trabalho anterior $^{13}$, condicionando a infestação inicial ou a reinfestação de ecótopos livres de triatomíneos e carreando pára eles a infecção tripanossômica.

Nos ecótopos artificiais o T.sordida prolifera bem, mostrando, porém, preferência pelos anexos domiciliários, especialmente galinheiros e abrigos de animais domésticos, embora invada também as habitações propriamente ditas. Aliás, nas áreas onde o P.megistus e sobretudo o T.infestans são espécies dominantes, a restrição ao peridomicílio é mais acentuada. Mas, com a eliminação daquelas espécies mediante o expurgo domiciliário, nota-se por parte do T.sordida agora livre de concorrência, uma acentuada tendência em invadir as habitações. Nos ecótopos artificiais o inseto, além de aves, suga o homem, os mamiferos domésticos, especialmente cães e gatos, os ratos comensais e animais sinantrópicos (morcegos e gambás), segundo as verificações de Barretto ${ }^{10}$ em 1968. Com freqüência se mostra parasitado pelo T.cruzi, exibindo porém índices de infecção inferiores aos do P.megistus, mas constitui elo importante no ciclo domiciliário de transmissão da infecção.

O Rhodnius prolixus, na Venezuela, oferece também um exemplo muito ilustrativo. Foi considerado, até há alguns anos, como espécie estritamente domiciliada ${ }^{41}$ até que os trabalhos de Gamboa ${ }^{49,50,51}$, Gomez-Nuñez ${ }^{53}$ e, mais recentemente, Pifano ${ }^{78}$ vieram demonstrar que ele prolifera abundamentemente em ecótopos silvestres, particularmente copas de diversas espécies de palmeiras e os grandes ninhos de garça, Mycteria americana.

Nos ecótopos naturais, segundo Pifano ${ }^{78}$, o R.prolixus convive com marsupiais, ratos silvestres e ratos comensais, além de aves e répteis, nos quais se alimenta, como demonstram os resultados de reações de precipitina. Encontrase, com freqüência, infectado, e constitui um elo importante no ciclo silvestre de transmissão do T.cruzi.

Ainda segundo Pifano ${ }^{78}$, o R.prolixus passou a se colonizar nas habitações humanas quando estas começaram a ser construídas nos focos naturais, utilizando-se folhas de palmeiras como material de cobertura. $O$ transporte do inseto para os ecótopos artificiais poude fazerse, assim, passivamente, como aliás demonstra a existência de casas já infestadas antes de serem habitadas, segundo Gamboa ${ }^{51}$. Mas, a migração ativa dos ecótopos naturais para os artificiais também ocorre, como indicam as investigações de Gomez-Nunẽz ${ }^{54}$; empregando exemplares marcados com $\mathrm{Co}^{60}$ verificou que eles se dispersam por seus próprios meios de locomoção, instigados principalmente pela estarvação, e, neste processo, invadem as habitações humanas e anexos.

Nos ecótopos artificiais, particularmente nas habitações humanas, o R.prolixus prolifera facilmente, sendo o homem a sua principal presa, segundo demonstram as reações de precipitina segundo Ponce, in Zeledon ${ }^{96}$. Mas convive também com animais domésticos e comensais e constitui o mais importante vector no ciclo domiciliário de transmissão do T.cruzi. 
Relativamente ao T.brasiliensis, no Nordeste do Brasil, e o P.pseudomaculata, no Brasil Central e no Nordeste do Brasil, faltam-nos dados mais detalhados sobre seu comportamento. Sabe-se no entanto, que são encontrados em habitações humanas e anexos, assim como em ecótopos naturais, o T.brasiliensis em locas de mocós $^{73}$ e o T.pseudomaculata em ocos de árvores e locas de animais ${ }^{7}$. 9 . Isso parece indicar que as espécies em questão são ubiquistas e têm papel tanto no ciclo silvestre quanto no ciclo domiciliário de transmissão do T.cruzi. É possivel que o comportamento do T.maculata, na Venezuela, seja semelhante, pois, embora freqüente em habitações humanas $e$ anexos ocorre também em ecótopos naturais, como demonstram as observações de Torrealba ${ }^{89}$, Torrealba e Diáz-Vásquez ${ }^{90}$ e Pifano ${ }^{78}$. Aliás, segundo Zeledón ${ }^{96}$ o T.maculata está exibindo tendência em substituir o R.prolixus no interior das habitações, quando este último é eliminado por desinsectização, tal como acontece no Brasil com o T.sordida ou P.megistus em relação ao T.infestans, como já discutimos.

o Triatoma quasayana, de ampla dispersão na Argentina, é uma espécie também muito ubiquista. Em natureza é encontrada em troncos de árvores, em cactáceas e sob pedras, convivendo com animais de sangue frio $^{28}$. Mas também é freqüente no peridomicílio, especialmente em galinheiros, pombais e currais. Segundo Abalos ${ }^{1}$, só os adultos invadem as habitações humanas nas noites quentes que prenunciam chuvas: mas Blaksley e Carcavallo ${ }^{28} \mathrm{em}$ 1968 afirmam que o T.quasayana reiteradamente é encontrado domiciliado em habitações humanas e que sua presença constitui um sério problema epidemiológico como possível substituto do T.infestans, quando este for eliminado pela desinsetização. Desde que sua infecção natural é freqüente, o T.quasayana, espécie ubiquista e em plena fase de adaptação aos ecótopos artificiais, parece ter papel importante em ambos os ciclos de transmissão do , T.cruzi, o silvestre e o domiciliário.

Ainda outras espécies que se criam tanto nos focos naturais quanto nos ecótopos artificiais são, segundo Zeledón ${ }^{95,96}$, o T.dímidiata e o T.phyllosoma no México e América Central, o R.pallescens no Panamá, etc.

\section{TRIATOMINEOS BEM ADAPTADOS AOS ECÓTOPOS ARTIFICIAIS MAS AINDA COM FOCOS RESIDUAIS SILVESTRES.}

Nesta categoria incluem-se os triatomíneos que, embora tenham conseguido adaptar-se com muito sucesso aos ecótopos artificiais, especialmente às habitações humanas, conservam ainda, pelo menos em alguns pontos de sua área de distribuição, resquícios de seus hábitos primitivos, sendo encontrados em focos silvestres residuais.

Zeledón 95,96 inclui nesta categoria duas espécies, o Rhodnius prolixus e o Triatoma infestans; mas, como vimos, a primeira, pelo menos na parte setentrional da América do Sul, deve ser considerada como indiferente quanto aos ecótopos naturais e artificiais.

Resta-nos, pois, como exemplo único e típico o Triatoma infestans, espécie extremamente bem adaptada às habitações humanas $e$ suas dependências e com uma longa história de relacionamento com o homem. Ocupa uma vasta área territorial na América do Sul e atinge, em certos pontos dessa área de distribuição, densidades altíssimas ${ }^{44}$. Tem marcada antropofilia, mas se alimenta também em mamfferos domésticos, especialmente cães e gatos, ratos comensais e mamíferos silvestres que invadem os ecótopos artificiais, especialmente gambás e morcegos, além naturalmente de aves domésticas, como demonstram as reações de precipiti$\mathrm{na}^{10,32,47,69}$. Dados os altos índices de infecção que pode apresentar, constitui o mais importante vector no ciclo domiciliário do T.cruzi em quase toda sua área de distribuição.

A despeito de achados de exemplares isolados ou pequenas colônias em ecótopos naturais, na Argentina, Bolívia e Paraguai ${ }^{5}, 27,70,92,94$, a generalidade dos autores tem insistido na estrita adaptação do T.infestans aos ecótopos artificiais, diante de resultados negativos obtidos em exaustivos inquéritos entomológicos em ecótopos naturais $2,38,46$.

No decurso de nossas investigações sobre triatomíneos do Vale do Rio Grande, Brasil ${ }^{20}$ tivemos a oportunidade de encontrar colônias maiores ou menores de T.infestans em ecótopos silvestres, em diversas localidades do Estado de São Paulo, confirmando as observacões anteriores de Barretto, Siqueira e Corrêa ${ }^{2}$, Barretto e Ferriolli $^{19}$ e Barretto ${ }^{6}, 10$. A maioria dos exemplares, muitos dos quais infectados, foi capturada em ocos e anfractuosidade do tronco de árvores, onde conviviam com gambás, ratos e morcegos, além de aves, como mostram as reações de precipitina. Exemplares menos numerosos foram èncontrados em palmeiras, convivendo com aves, ratos e morcegos.

Segundo alguns autores, especialmente Aba los e Wygodzinsky ${ }^{2}$, em 1951, esses criadouros não seriam focos residuais naturais e sim focos 
antropúrgicos, resultantes da invasão por exemplares de T.infestans provenientes de habitações humanas e anexos situados nas vizinhanças; a presença dos triatomíneos nesses focos seria, em realidade, uma extensão da infestação domiciliária. É possivel que em alguns casos isso aconteça, embora nossas observações indiquem que o T.infestans não manifeste tendência de migrar, por atividade própria, de um ecótopo para outro, sendo sua dispersão passiva e resultante do transporte pelo próprio homem. Seja como for, temos dados que mostram a existência de focos silvestres em áreas desabitadas, focos esses sem a menor conexão com as habitações humanas mais próximas, aliás situadas, muitas vezes, a uma distância de muitos quilômetros. Nossa opiniăo a respeito desses focos concorda, pois, com as de Bejarano ${ }^{25,26}$.

Assim, o T.infestans, em determinadas circunstâncias pode colonizar-se em ecótopos na turais, constituindo um elo no ciclo silvestre do T.cruzi, transmitindo o parasita entre gambás, ratos e morcegos com os quais convive.

\section{TRIATOMINEOS COMPLETAMENTE DO- MICILIADOS}

Este grupo inclui os triatomíneos que, até agora, têm sido encontrados exclusivamente em ecótopos artificiais, mais especialmente em ha bitações humanas. Parece que a única espécie nestas condições é o Triatoma rubrofasciata.
Trata-se de uma espécie cosmopolita, aparentemente originária da Ásia, que vive nas habita ções' humanas em cidades litorâneas. Referências mais antigas sobre seu encontro em cidades do interior devem ser tomadas com cautela, mas as observações mais recentes de Lucena e Marques $^{66}$ em 1955, em localidades da Zona da Mata, no interior de Pernambuco, Brasil, não deixam dúvidas.

Segundo alguns autores ${ }^{77}$ o T.rubrofasciata convive com ratos comensais nos telhados e sótãos das casas, sendo responsável pela transmissão do T.cruzi entre esses animais ${ }^{39}, 57$. sem contudo entrar em contato Intimo com o homem. Dal ser considerado de pequena importấncia epidemiológica.

Ainda segundo Pessoa ${ }^{77}$, o T.rubrofasciata, descendo de seus ninhos preferidos, pode invadir os aposentos das habitações e transmitir o T.cruzi ao homem, uma vez que sua infecção natural já foi repetidamente comprova$\mathrm{da}^{39,43,66,85}$. Esta hipótese, aliás, é mais recentemente comprovada por Becerra, Coura e Ferreira ${ }^{24}$, em 1970, que observaram exemplares do triatomíneos alimentando-se no homem no Rio de Janeiro, Brasil. Assim, o T.rubrofasciata, aliado a outras espécies domiciliárias, pode constituir um elo no ciclo de transmissão domiciliário do T.cruzi, como já haviam assinalado Lucena e Marques ${ }^{6}$. $^{\circ}$

\section{SUMMARY}

On the basis of their ecological behavior the triatomines are distributed into six groups:

1. Typically wild insects, $i$. e., species that are found only in natural ecotopes, never being collected in human dweellings and their annexes. Consequently they never come into contact with man and domestic, commensal or synantropic mammals, unless they enter the natural foci. But these insects may have a more or less important role in maintaining the wild trypanosome enzooty. Examples: Psammolestes coreodes, Psammolestes tertius, Cavernicola pilosa, Triatoma dispar, Triatoma delpontei and other little known species.

2. Essentially wild insects whose adults occasionally invade human dwellings and their annexes but are unable to thrive in artificial ecotopes. In addition to the role in the wild cycle of transmission of T.cruzi, they may come into contact with man and domestic or domiciliated mammals and transmit the infection both in natural foci and artificial ecotopes. Examples: Panstrongylus geniculatus, Triatoma rubrovaria, Triatoma arthurneivai, Triatoma patagonica, Triatoma eratyrusiforme, Rhodnius domesticus, etc. 
3. Wild insects beginning to adapt to artificial ecotopes where they give rise to small colonies spesially in animal dens, and, more rarely, inside human dwellings. In addition to the role in the wild cycle of transmission of T.cruzi, they may convey the parasite from natural to artificial ecotopes and, under certains circunstances, participate of the domiciliary cycle of T.cruzi. Examples: Rhodnius neglectus, Triatoma vitticeps, Triatoma platensis, etc.

4. Insects that breed indifferently in natural or artificial ecotopes. A/though they have succeded in adapting themselves to human dwellings and domestic animal dens, they are also found in many natural ecotopes. In addition to the role in the wild transmission cycle of T.cruzi they are important in the conveyance of the parasite from natural foci to artificial ecotopes and in the inicial infestation or in the reinfestation of these ecotopes, and are an efficient ring in the domiciliary chain of transmission of T.cruzi. Examples: Panstrongylus megistus, Rhodnius prolixus, Rhodnius pallescens, Triatoma sordida, Triatoma brasiliensis, Triatoma pseudomaculata, Triatoma quasayana etc.

5. Insects well adapted to artificial ecotopes, but sometimes still found in residual natural breeding places. They are most important in the domiciliary cycle of transmission of T.cruzi, but may play, at least in certain areas, a small role in the wild cycle of transmission of the infection. Example: Triatoma infestans.

6. Entirely domiciliated insects. In spite of being found only in houses, they seem to have a minor role in the transmission of T.cruzi to man, because they live more into contact with commensal rats, although thay may also bite man. Example: Triatoma rubrofasciata.

\section{REFERENCIAS BIBLIOGRÁFICAS}

1. ABALOS, J.W. - Distribución de vectores en Argentina. Simposio Internacional sobre Enfermedad de Chagas, Buenos Aires, pgs. 347-356, 1972.

2. ABALOS, J.W. \& WYGODZINSKY,P. Las Triatominae Argentinas (Reduviidae, Hemipterae), Monogr. 2 Inst. Med. Reg, Tucuman, 179 pgs., 1951.

3. ALMEIDA, F.B. - Triatomineos da Ama zônia. Encontro de três espécies naturalmente infectadas por Trypanosoma semeIhante ao cruzi no Estado do Amazonas (Hemiptera, Reduviidae). Acta Amaz. 1: 89-93, 1970.

4. ALVES, U.P. \& NODA, J. - Os transmissores da doença de Chagas na Região de Sorocaba, Estado de São Paulo, Brasil, Arq. Hig. Saúde Públ., 29: 141-157, 1964.

5. BARRERA, J.M. - Contribución al conocimiento de la peste silvestre en la Argentina. Caracteres del brote de Mendonza en 1937. Rev. Inst. Bacteriol. Malbrán, 8: 431-454, 1939.

6. BARRETTO, M.P. - Aspectos da epidemiologia da tripanossomose americana, infeccão com focos naturais, com especial refe- rência à Região Nordeste do Estado de São Paulo. Tese Prof. Cat. Fac. Farm. Odontol. Ribeirão Preto (mimeografada). 139 pgs., 1966.

7. BARRETTO, M.P. - Estudos sobre reservatórios e vectores silvestres do Trypanosoma cruzi. XVII. Contribuição para o estudo dos focos naturais da tripanossomose ame ricana, com especial referência à Região Nordeste do Estado de São Paulo, Brasil, Rev. Soc. Bras. Med. Trop., 1-23-25, 1967.

8. BARRETTO, M.P. - Estudos sobre reservatórios e vectores silvestres do Trypanosoma cruzi. XXI: Observações sobre a ecologia do Panstrongy/us geniculatus (Latreille, 1811). Rev. Brasil. Biol., 27: 337-348, 1967.

9. BARRETTO, M.P. - Estudos sobre reservatórios e vectores silvestres doTrypanossoma cruzi. XIX - Inquérito preliminar sobre triatomíneos silvestres no Sudoeste do Estado de Goiás, Brasil (Hemiptera, Reduviidae). Rev. Inst. Med. Trop. São Paulo, 9: 313-320, 1967.

10. BARRETTO, M.P. - Estudos sobre reservatórios e vectores silvestres do Trypanosoma 
cruzi. XXXI: Observações sobre a associação entre reservatórios e vectores, com especial referência à Região Nordeste do Estado de São Paulo. Rev. Brasil, Biol., 28: 481-494, 1968.

11. BARRETTO, M.P. - Estudos sobre reservatórios e vectores silvestres do Trypanosoma cruzi. XLV: Inquérito preliminar sobre triatomíneos silvestres no Sul de Mato Grosso, Brasil (Hemiptera, Reduviidae). Rev. Brasil. Biol., 31: 225-233, 1971.

12. BARRETTO, M.P. - Nuevos conceptos ecoIógicos en la epidemiologia de la Enfermedad de Chagas. Mem. II Congr. Centroamer. y I Nac. Microbiol., Panama. 131-147, 1971.

13. BARRETTO, M.P. - Possible role of wild mammals and triatomines in the transmission of Trypanosoma cruzi to man. Proc. Internat. Symposium on New Approaches in American Trypanosomiasis Research, Belo Horizonte, 1975. Washington: Pan American Helath Organization, pp. 307-316, 1976.

14. BARRETTO, M.P. \& ALBUQUERQUE, R.D.R. - Estudos sobre reservatórios e vectores silvestres do Trypanosoma cruzi. XXXIII - Infecção experimental e natural do Psammolestes tertius Lent \& Jurberg, 1965 pelo T.cruzi. Rev. Inst. Med. Trop. São Paulo, 11: 165-168, 1969.

15. BARRETTO, M.P., ALBUQUERQUE, R.D. \& FUNAYAMA, G.K. - Estudos sobre reservatórios e vectores silvestres do Trypanosoma cruzi. XXXVI: Investigações sobre triatomíneos de palmeiras no Municipio de Uberaba, MG., Brasil. Rev. Brasil. Biol., 28: 577-588, 1969.

16. BARRETTO, M.P. \& CARVALHEIRO, J.R. - Estudos sobre reservatórios e vectores silvestres do Trypanosoma cruzi. XII. Inquérito preliminar sobre triatomíneos silvestres no Município de Uberaba, Minas Gerais, Rev. Brasil. Biol., 26: 5-14, 1966.

17. BARRETTO, M.P. \& CARVALHEIRO,J.R. - Estudos sobre reservatórios e vectores silvestres do Trypanosoma cruzi. XVIII. Observações sơbre ecologia do Psammolestes tertius Lent e Jurberg, 1965 (Hemiptera, Reduviidae) Rev. Brasil. Biol., 27: 13-25, 1967.
18. BARRETTO; M.P. \& CARVALHEIRO, J.R. - Estudos sobre resenvatórios e vectores silvestres do Trypanosoma cruzi. XXV: Observações sobre a infestação de ninhos de Anumbius annumbi Vieillot, 1817 e de Phacellodomus rufifrons rufifrons (Wie, 1821) pelo Psammolestes tertius Lent \& Jurberg, 1965 (Hemiptera, Reduviidae). Rev. Brasil. Biol., 28: 11-17, 1968.

19. BARRETTO, M.P. \& FERRIOLI FO, F. Estudos sobre reservatórios e vectores silvestres do Trypanosoma cruzi. IV. Infeçãa natural do Triatoma infestans, encontrado em ecótopos silvestres, por tripanossomo semelhante ao T.cruzi. Rev. Inst. Med. trop. São Paulo, 6:219-224, 1964.

20. BARRETTO, M.P., SIQUEIRA, A.F., CARVALHEIRO, J.R. FERRIOLLI FO, F. \& FERNANDES, L.A.R. - Estudos sobre reservatórios e vectores silvestres do Trypanosoma cruzi. LIX. Distribuição geográfica de triatomineos na Bacia Hidrográfica do Rio Grande, Brasil (Hemiptera, Reduviidael. Rev. Brasil, Biol., 35:21-30, 1975.

21. BARRETTO, M.P., SIQUEIRA, A.F. \& CORREA, F.M.A. - Estudos sobre reservatórios e vectores silvestres do Trypanosoma cruzi. 1. Encontro do Triatoma infestans (Hemiptera, Reduviidae) em ecótopos silvestres. Rev. Inst. Med. Trop. São Paulo, 5: 289-293, 1963.

22. BARRETTO, M.P., SIQUEIRA, A.F., FERRIOLLI Fo F. \& CARVALHEIRO, J.R. - Estudos sobre reservatórios e vectores silvestres do Trypanosoina cruzi. XXIII - Observações sobre criadouros de Rhodnius neglectus Lent, $1954 \mathrm{em}$ bibtopos artificiais (Hemiptera, Reduviidae). Rev. Inst. Med. Trop. São Paulo, 10: 163-170, 1968.

23. BARRETTO, M.P., SIQUEIRA, A.F. \& FREITAS, J.L.P. - Estudos sobre reservatórios e vectores silvestres do Trypananosoma cruzi. II. Encontro do Panstrongylus megistus em ecotopos silvestres no Estado de São Paulo (Hemiptera, Reduviidae). Rev. Inst. Med. Trop. São Paulo, 6:56-63, 1964.

24. BECERRA FUENTES, F., COURA, J.C. \& FERREIRA, L.F. - Observações sobre o Triatoma rubrofasciata (Der Geer 1773) no Estado da Guanabara. Rev. Soc. Brasil. Med. Trop., 5: 47-54, 1971. 
25. BEJARANO, J.F.R. - Estado selvático de T.infestans $y$ otros aspectos a tener en cuenta para la eliminación de la enfermedad de Chagas. 2a Jorn. Entomoepidemil. Argent. 3: 171-196, 1967.

26. BEJARANO, J.F.R. - Importância del estado selvage de Triatoma infestans Klug, 1834 (Reduviidae, Hemiptera) en la lucha contra la enfermedad de Chagas. Comun, 1 er Congr. Argent. Parasitol., Buenos Aires, 1972.

27. BERG, G. - Hemiptera Argentina. Ensayo de uma monografia de los hemíteros heterópteros de la República Argentina. An. Soc. Cient. Argentina, 7: 262-278, 1879.

28. BLASKLEY, J.C. \& CARCAVALLO, R.V. - La enfermedad de Chagas - Mazza en la Argentina. Buenos Aires, 142 pgs., 1968.

29. BRIONES, S. - Xenodiagnóstico para enfermedad de Chagas en ninõs de la Gobernación de la Pampa. An. Inst. Med. Reg. Tucuman, 3: 147-152, 1951.

30. CARVALHO, A.C. \& VERANO, O.T. Epidemiologia e profilacia da doença de Chagas em Goiás. Rev. Goiana Med., 2: 241-277, 1966.

31. CORREA, F.M.A., CARVALHEIRO, J.R. \& BARRETTO, M.P. - Estudos sobre reservatórios e vectores silvestres do Trypanosoma cruzi. V. Observações sobre a ecologia e a morfologia do Psammolestes coreodes Bergroth, 1911 (Hemiptera, Reduviidae). Rev. Brasil. Biol., 24: 259-268, 1964.

32. CORREA, R.R. \& AGUIAR, A.A. - O teste da precipitina na identificação da fonte alimentar do Triatoma infestans $(\mathrm{He}-$ miptera, Reduviidae). Arq. Hyg. Saúde Públ., 17: 3-8, 1952.

33. CORRÉA, R.R., ALVES, UP. \& NODA, J. - Presença do Triatoma arthurneivai em Sorocaba (Hemiptera, Reduviidae). Rev. Paulista Med., 6: 267- 1962.

34. CORREA, R.R., ALVES, U.P. \& NODA, J. - Nota sobre o Triatoma arthurneivai. Seu criadouro extradomiciliar (Hemiptera, Reduviidae). Rev. Brasil. Malariol. D. Trop., 17:217-234, 1965.

35. CORREA, R.R. \& FERREIRA, O.A. Distribuição geográfica, habitats e infecção do Triatoma sordida (Hemiptera, Reduvii- dae) no Estado de São Paulo. Rev. Inst. Med. Trop. São Paulo, $1: 207-213,1959$.

36. CORREA, R.R, LIMA A.R. - Nota sobre o gênero Rhodnius Stal, 1859, no Estado de São Paulo, Brasil (Hemiptera; Reduviidae, Triatomianae). Arg. Hig. Saúde Públ., 18:267-280, 1953.

37. CORREA, R.R., SILVA, E.O.R. \& SCHIA VI, A. - Observações sobre o Panstrongy. lus megistus, transmissor da moléstia de Chagas (Hemiptera, Reduviidae). Arq. Hig. Saúde Públ., 18:267-280, 1953.

38. CORREA, R.R., SILVA, T.L. \& RAMOS, A.S. - Os triatomíneos vectores da moléstia de Chagas no Estado de São Paulo, Brasil. Arq. Hig. Saúde Públ., 18:535-546, 1953.

39. COSTA, A. - Triatomíneos e tripanossomas por eles veiculados na zona central da Cidade de Salvador. Tese Prof. Catedr. Fac. Med. Univ. Bahia, Salvador, 81 págs., 1955.

40. COUTINHO, P.P., PINTO, O.S. \& BARBOSA, J.C. - Contribuição ao conhecimento da distribuição dos triatomíneos domiciliares e de seus índices de infecçăo pelo $T$. cruzi no Estado de Rio Grande do Sul, Brasil. An. IX Congr. Brasil. Hyg., Porto Alegre, 1951, pgs. 239-252, 1952.

41. COVA-GARCIA, P. \& SUAREZ, M.A. Estudo de los triatomíneos em Venezuela. Publ. Div. Malariol., no 11, 209 pgs., 1959.

42. DIAS, E., MELLO, J.B., COSTA, O., DAMASCENO, R. \& AZEVEDO, M. - Investigações sobre esquizotripanose de morcegos no Estado do Pará; encontro do barbeiro Cavernicola pilosa como transmissor. Rev. Brasil. Biol., 2:103-110, 1942.

43. DIAS, E. \& NEVES, O. - Determinação da infecção natural por Schizotrypanum em T. rubrofasciata no Estado de Pernambuco, Mem. Inst. O. Cruz: 331-334, 1943.

44. DIAS, E. \& ZELEDON, R. - Infecção domiciliária em grau extremo por Triatoma infestans. Mem. Inst. O. Cruz, 53:473-486, 1955.

45. FORATTINI, O.P., JUAREZ, E. \& RABELLO, E.X. - Dados sobre a biologia do Triatoma arthurneivai no Sudeste do Estado de São Paulo, Brasil (Hemiptera, Reduviidae). Rev. Saúde Públ., 2:186-193, 1968. 
46. FREITAS, J.L.P. - Importância do expurgo seletivo dos domicílios e anexos para a profilaxia da moléstia de Chagas pelo combate aos triatomíneos. Arq. Hig. Saúde Públ., 28:217-272, 1963.

47. FREITAS, J.L.P., SIQUEIRA, A.F. \& FERREIRA, O.A. - Investigações epidemiológicas sobre triatomíneos de hábitos domésticos e silvestres com auxflio da reação de precipitina. Rev. Inst. Med. Trop. São Paulo, 2:90-99, 1960.

48. GALINDO, P. \& FAIRCHILD, G.B. Notes on the habits of two blood suking bugs, Triatoma dispar Lent., 1950 and Eratyrus cuspidatus Stal, 1859 (Hemiptera, Reduviidae). Proc. Entomol. Soc. Wash., 64:229-230, 1962.

49. GAMBOA, C.J. - Comprobación de Rhodnius prolixus extradomésticos. Bol. Inform. Dir. Màlariol. San. Amb., 5:139-142, 1961.

50. GAMBOA, C.J. - Dispersión de Rhodnius prolixus en Venezuela. Bol. Inform. Dir. Malariol. San. Amb., 3:262-273, 1962.

51. GAMBOA, C.J. - Comprobación de Rhodnius prolixus extradomiciliario en Venezuela. Bol. Ofic. Sanit. Panam., 54:18-25, 1963.

52. GOMES, J.F. - Triatoma e moléstia de Chagas no Estado de São Paulo. I. Congresso Med. Paulista, 1916, 2:193-214, 1917.

53. GÓMEZ-NUNẼZ, J.C. - Notas sobre la ecologia del Rhodnius prolixus. Bol. Inform. Dir. Malariol. San. Amb., 3:330-335, 1963.

54. GÓMEZ-NUNẼZ, J.C. - Resting places, dispersal and survival of $\mathrm{Co}^{60}$ tagged adult Rhodnius prolixus. J. Med. Entomol., 6:83-86, 1969.

55. GUERRERO, L., GARCIA MATÍN, G. \& DOMINGUES QUESADA, M. - Campaña contra la Enfermedad de Chagas. Kasmera, 2:47-97, 1965.

56. LEAL, H., FERREIRA NETO, J.A. \& MARTINS, C.M. - Dados ecológicos sobre triatomíneos silvestres na liha de Santa Catarina, Brasil. Rev. Inst. Med. Trop. São Paulo, 3:213-220, 1961.

57. LEAL, J.M., SHERLOCK, J.A. \& SERAFIM, E.M. - Observações sobre o combate aos triatomíneos domiciliários com BHC em Salvador, Bahia. Rev. Brasil. Malariol. D. Trop. 17:65-73, 1965.
58. LENT, H. - Observações sobre a fauna de alguns ninhos de aves. Bol. Biol., n.s., 4:258-260, 1939.

59. LENT, H. - Transmissores da moléstia de Chagas no Estado do Rio de Janeiro, Res. Flum. Med., 7:151-161, 1942.

60. LENT, H. - O gênero Rhodnius Stal, 1859 (Hemiptera, Reduviidae). Rev. Brasil. Biol., 8:297-339, 1948.

61. LENT, H. - Comentários sobre o gênero Rhodnius Stal, com descrição de uma nova espécie do Brasil (Hemiptera, Reduviidae). Rev. Brasil., 14:237-247, 1954.

62. LENT, H. \& JURBERG, J. - O gênero Psammolestes Bergroth, 1911, com um estudo sobre a genitália das espécies (Hemiptera, Reduviidae). Rev. Brasil. Biol., 25:349-376, 1965.

63. LENT, H. \& MARTINS, A.V. - Estudos sobre os triatomíneos do Estado de Minas Gerais, com descrição de uma espécie nova. Rev. Emtomol., 11:877-886, 1940.

64. LUCENA, D.T. - Ecologia dos triatomfneos do Brasil. Rev. Brasil. Malariol. $D$. Trop., 11:577-635, 1959.

65. LUCENA, D.T. \& LUCENA, N.T. - Psammolestes coreodes Bergroth, 1911 (Hemiptera, Reduviidae) em Pernambuco, Brasil, Rev. Inst. Med. Trop. São Paulo, 7:160-168, 1965.

66. LUCENA, D.T. \& MARQUES, R.J. Subsídio para o estudo ecológico do Triatoma rubrofasciata no Brasil. An. Fac. Med. Univ. Recife, 15:19-31, 1955.

67. MARQUES, P.A.A., CONESSA, P.S., NAKAMURA, S. SANTOS, J. \& SANTANA, R. - Achados de Rhodnius neglectus Lent, 1954 em domicílios e anexos. Rev. Goiana Med., 7:63-70, 1961.

68. MARTÍNEZ, A. \& CICHERO, J.A. - Los vectores de la Enfermedad de Chagas, Argentina, 56 pags., 1972.

69. MAYER, H.F. \& ALCARAZ, I.L. - Estudos relacionados con las fuentes alimentares de Triatoma infestans (Hem., Reduviidae). An. Inst. Med. Reg., 4:195-201, 1955.

70. MAZZA, S. - Comprobaciones de Triatoma platensis, Eutritoma osvaldoi, Panstrongylus seai y Psammolestes coreodes en la Provincia de Santiago del Estero, todas 
ellas sin infestación, y Etriatoma sordida con infestación per el $S$. cruzi. Otros datos sobre infestación esquizotripanósica natural silvestre de Triatoma infestans. Prensa Med. Argent., 30:1583-1590, 1943.

71. MILES, M.A. - Distribution and importance of triatominae as vectors of Trypanosoma cruzi. Proc. Intern. Symposium on New Approaches in American Trypanosomiasis Research, Belo Horizonte, 1975. Washington: Pan American Health Organization, pp. 48-53, 1976.

72. MINTER, D.M. - Feeding patterns of some triatomine vector species. Proc. Intern. Symposium on New Approaches in American Trypanosomiasis Research, Belo Horizonte, 1975. Washington: Pan American Health Organization, pp. 33-46, 1976.

73. NEIVA, A. - Contribuição para o estudo dos Reduviidae hematófagos. Mem. Inst. $O$. Cruz, 6:35-39, 1914.

74. NEIVA, A. \& PINTO, C. - Estado actual dos conhecimentos sobre o gênero Rhodnius Stal, com a descrição de uma nova espécie. Brazil-Med., 37:20-24, 1923.

75. OLIVEIRA, G. - Isolamento do Trypanosoma cruzi e outras noções concernentes à moléstia de Chagas no Rio Grande do Sul (Nota prévia). Brazil-Med., 34:142-143, 1920.

76. PELLEGRINO, J. Transmissores da doença de Chagas no Estado de Minas Gerais. Rev. Assoc. Med. Minas Gerais, 2:43-66, 1951.

77. PESSOA, S.B. - Domiciliação de triatomineos e epidemiologia da doença de Chagas. Arq. Hig. Saúde Públ., 27:161-171, 1962.

78. PIFANO, C., F: - La dinámica epidemiológica de la Enfermedad de Chagas en el Valle de los Naranjos, Estado Carabobo, Venezuela. I. Contribución al estudio de los focos naturales silvestres del Schizotrypanum cruzi Chagas, 1909. Arch. Venez. Med. Trop. Parasitol. Med., 5:3-29, 1973.

79. PINTO, A.F.S., SANTOS, U.M., ZAGANELLI, F.L., ALMEIDA, A.Z. \& CARRACHO, P.V. - Doença de Chagas no Estado do Espirito Santo. VI. Observações sobre o Triatoma vitticeps, vector do Trypanosoma cruzi no Município de Alfredo Chaves, E.S. Rev. Soc. Brasil Med. Trop. 3:53-54, 1969.
80. PINTO, C. - Tripanosomiasis cruzi (Doença de Chagas) no Rio Grande do Sul, Brasil. Mem. Inst. O. Cruz, 37:443-537, 1942.

81. PINTO, C. - Epidemiologia da doença de Carlos Chagas no Estado do Rio Grande do Sul, Brasil. Mem. Inst. O. Cruz, 44:363-400, 1946.

82. PRIMIO, R. - Distribuição geográfica dos triatomíneos no Rio Grande do Sul. An. $X$ Congr. Brasil. Hig. (Belo Horizonte, 1952). pgs. 440-449, 1953.

83. PRIMIO, R. - Atual distribuição geográfica dos triatomíneos e seus indices de infecção no Rio Grande do Sul. An. Fac. Med. Porto Alegre, 17:17-37, 1957.

84. SANTOS, U.M., PINTO, A.F.S., ALMEIDA, A.Z., ZAGANELLI, F.L., CARRAN. CHO, P.V. \& NETTO, A.N. - Doença de Chagas no Estado do Espírito Santo. III. Vectores do tripanosoma. Rev. Soc. Bras. Med. Trop. 3:51-52, 1969.

85. SHERLOCK, I.A. \& SERAFIM, E.M. Fauna Triatominae do Estado da Bahia, Brasil. VI - Prevalência geográfica da infecção dos triatomíneos por $T$. cruzi. Rev. Soc. Brasil. Med. Trop., 8:129-142, 1974.

86. SOUSA, O.E. \& GALINDO, P. - Natural infections of Triatoma dispar Lent, 1950 with Trypanosoma cruzi in Panama. Amer. J. Trop. Med. Hyg., $21: 293-295,1972$.

87. TALICE, R.V., COSTA, R.S., RIAL, B. \& OSIMANI, J.J. - Los 100 primeros casos agudos confirmados de enfermedad de Cha gas (Trypanosomiasis americana) en el Uruguay. Montevideo: A. Monteverde Ed., 249 pgs. 1940

88. TORANZOS, L.B. - La infestación de Triatoma (Eutriatoma) rubrovaria por el Schizotrypanum cruzi. Su comprabación en la República Argentina. Rev. Inst. Med. Regional, 1:105-116, 1944.

89. TORREALBA, J.F. - Consideraciones sobre la enfermedad de Chagas en Zaraza. Gac. Med. Caracas, 42:356-361, 1935.

90. TORREALBA，J.F. \& DIÁZ-VÁSOUEZ, A. - Una pequeña contribución al estudio de focos extradomésticos de triatomíneos transmissores de la enfermedad de Chagas en Venezuela. Gac. Med. Caracas, $61: 259-267,1953$. 
91. TORRES, M. - Alguns fatos que interessam à epidemiologia da moléstia de Chagas. Mem. Inst. O. Cruz, 7:120-128, 1915.

92. TORRICO, R.A. - Hallazgo de Eratyrus mucronatus. Infestación natural de Vinchucas de cerro y Triatoma sordida en Cochabamba. An. Lab. Central, 1:19-23, 1946.

93. TRAVASSOS, L \& FREITAS, J.F.T.-Relatório de sétima excursão científica do Instituto Osvaldo Cruz, realizada à zona da Estrada de Ferro Noroeste do Brasil, em Maio de 1942. Mem. Inst. O. Cruz, 38:385-412, 1943.

94. VELÁZOUEZ, C.J. \& GONZÁLEZ, G. -
Aspectos de la enfermedad de Chagas en el Paraguay. Rev. Guiana Med., 5:357-373, 1959.

95. ZELEDÓN, R. - Los vectores de la enfermedad de Chagas en America. Simposio Internat. Enfermedad de Chagas, Buenos Aires, pgs. 327-345, 1972.

96. ZELEDÓN, R. - Epidemiology, modes of transmission and reservoir hosts of Chagas' disease. Symposium on Trypanosomiasis and Leishmaniasis, with special reference to Chagas' disease. (Ciba Foundation Symposium). Excerpta Med., 20'(N.S.): 51-77, 1974. 